\title{
STRESS AND MUSCLE DAMAGE MONITORING IN HIGH-LEVEL BASKETBALL PLAYERS
}

\author{
AUDRIUS GOCENTAS ${ }^{1}$, ANATOLI LANDÕR ${ }^{2}$ \\ ${ }^{1}$ Lithuanian University of Educational Sciences, Lithuania \\ ${ }^{2}$ Faculty of Medicine, University of Tartu, Estonia
}

\section{ABSTRACT}

Close complex monitoring during the entire training period is essential for professional athletes to assess the effect of the training programme and to determine the quality of recovery. Follow-up of biochemical and hormonal variables throughout the season can help realize the abovementioned goals.

Objectives: To determine baseline blood creatine kinase (CK) and cortisol (C) levels in professional basketball players before the beginning of regular training and to follow up the subsequent levels of blood $\mathrm{C}$ and $\mathrm{CK}$ over an entire season with analysis of dynamic changes in relation to individual coefficients of variation.

Methods: Six professional basketball players were monitored from the first pre-season day to the end of a regular season. Blood samples were collected at six time points: before the pre-season, after the pre-season and four times during the in-season to determine blood $\mathrm{C}$ and $\mathrm{CK}$ concentrations.

Results: Individual trends of the investigated variables were established and analysed with respect to the calculated intra- and inter-individual coefficients of variation for $\mathrm{C}$ and $\mathrm{CK}$. Dynamic changes in $\mathrm{C}$ and $\mathrm{CK}$ levels coincided with changes in the content of the training process.

Conclusion: In summary, the findings in the present study suggest that long-term intensive exercise in basketball has a significant influence on the blood cortisol profile. Muscle damage was associated with bodily collisions between the players but not with stress response in the current study.

Key words: stress, cortisol, monitoring, basketball 


\section{INTRODUCTION}

The goal of athletic training is to prepare the body for an intensive stimulus, to increase the capacity of the various systems to carry strenuous workloads, and hence to enhance performance. Because of the increased training loads and the growing number of matches accompanying modern team game sports, it is important to find simple, rigorous, and regular monitoring tools to prevent injury, to stimulate recovery, and to optimally train athletes. Various biochemical, hormonal and immune markers, were selected to evaluate the athletes' physical status and to recognize overreaching and overtraining during a training programme with different degrees of success. However, the lack of specific and/or universal markers has still complicated detection of fatigue [4, $8,9,14,21]$.

Physical stress as a consequence of the training loads triggers a number of physiological changes such as activation of the sympathetic nervous system and the hypothalamic - pituitary - adrenal axis [5,24]. The testosterone to cortisol $(\mathrm{T} / \mathrm{C})$ ratio has been suggested as simplified marker for assessing the balance between anabolic and catabolic processes [17]. In our previous study we showed that changes in the $\mathrm{T} / \mathrm{C}$ ratio mostly depended on the value of cortisol in professional basketball players [11]. Cortisol is considered an important stress hormone which acts to mediate catabolic activity, decreasing protein synthesis and increasing protein degradation in the muscle tissue [5, 17, 24]. Blood CK level is considered an indirect measure of skeletal-muscle damage in response to the physical load $[2,6,18,19,23]$. Higher intensity and total volume as well as short rest intervals are associated with the magnitude of stress and muscle damage $[2,9,19,23]$. Numerous laboratory and field studies address changes in blood cortisol as response to different loads in different environments among athletes $[1,3,4,6,8,10,16,18,21,23]$. Recently Cunniffe and al. [6] described in detail "pericompetitional" fluctuations in the blood level of cortisol and creatine kinase in response to high-level rugby match. Despite the informative content, frequent interventions have met limited practical application in competitive sport because of possible alteration in the regular course of the training cycle. Standardised repeated single interventions as longitudinal measures of hormonal, immunological and biochemical variables have been described in different sports, including team-game sports, as an expression of the impact of training [3, 13, 15, 16, 18-20, 23]. Analysis of longitudinal measurements can be carried out in comparison with an accepted reference interval but such an approach is not always productive in the case of athletes because of biological, procedural and environmental influences [12]. 
An alternative way to test the difference between longitudinal measures is evaluation of individual dynamic changes in relation to an intra-individual coefficient of variation. Such analysis can be used in the case of absence of a normative reference interval. This method could also be applied when changes in serial testing do not exceed normal limits for the reference population [22].

Data about dynamic changes in hormonal and biochemical variables in relation to the training process in basketball are still scarce. Martinez et al. [20] described the course of biochemical and hormonal variables through the main time points of a basketball macro cycle starting from the pre-season. However, inclusion of more time points throughout the season in analysis would give more reliable results. We aimed to determine baseline blood C and CK levels in professional basketball players before the beginning of regular training and to follow the subsequent levels of blood C and CK over an entire season with analysis of dynamic changes in relation to individual coefficients of variation.

\section{MATERIAL AND METHODS}

\section{Subjects}

The athletes of the Lithuanian Basketball League, i.e. representatives of the same club, were recruited in this study. Some players of this team were excluded because of delayed joining the team practices or because of transfers. Finally, the study was conducted on six male international-level basketball players throughout the pre-season and the whole regular season. All subjects were informed about the purpose of the study and written informed consent was obtained according to the Declaration of Helsinki. The participants were aware that all the individual study results would remain confidential. Prior to the study, the players underwent a physical examination by a medical staff, and were cleared of any medication or diseases that might confound or limit their ability to fully participate in the investigation. The physical characteristics of the participants were the following: age (years) $24.3 \pm 3.22$, body mass $(\mathrm{kg}$ ) $98.7 \pm 13.79$, height $(\mathrm{cm}) 200.2 \pm 4.62$, and peak oxygen consumption $(\mathrm{ml} / \mathrm{kg} / \mathrm{min}) 50.8 \pm 6.06$ as mean $( \pm \mathrm{SD})$. Experimental procedures

All involved athletes were observed from the first day after the summer vacation (mid-August) until the end of a regular season (mid-April). They had been recruited for Euroleague matches as well for Lithuanian and Baltic championships matches. 
The period of observation comprised 228 practices, 71 matches and 38 rest days. The $\mathrm{C}$ and $\mathrm{CK}$ concentrations in rest conditions were measured at six time points. The first blood sample (M1) was taken before the first practice after the off-season and represented the rest reference value. The second sampling (M2) took place during pre-season preparation (September). All other consecutive samplings took place throughout the in-season (October=M3, December $=M 4$, February $=$ M5 and April=M6). The last sampling took place after the end of the regular season and 5-day tapering before playoffs. To avoid any confounding effects of variations in circadian rhythm and food intake on hormonal secretion, the athletes provided blood samples at same time (09.00-09.30) after overnight fasting. All samplings, except for the first one, took place $14 \mathrm{~h}$ after the last physical load. The analysed parameters were determined by standard clinical laboratory techniques. All venous blood samples were drawn via antecubital venipuncture in a semirecumbent position. Blood was collected in Vacutainer tubes. Blood C concentrations were assayed using an Immulite 2000 analyser and an immunoassay (Siemens Healthcare Diagnostics Products Ltd., Llanberis, Gwynnedd, UK) according to the manufacturers' instructions. The CK activity was assessed with an Advia 1650 analyser (Siemens Healthcare Diagnostics, Deerfield, IL, USA) using the IFCC method and according to the manufacturer's instructions. All samples were tested in the same series to avoid inter-assay variations.

\section{Statistics}

The data were analysed using SPSS for Windows (version 13.0; SPSS Inc., Chicago, IL) and expressed as a mean \pm standard deviation. The coefficient of variation $(\mathrm{CV})$ was calculated as the ratio of mean to standard deviation. The difference in level of cortisol between two consecutive measurements was expressed as proportion $\left(\mathrm{C}_{\text {current }}-\mathrm{C}_{\text {previous }}\right) / \mathrm{C}_{\text {current }}$. The Pearson correlation coefficient was used to assess possible relationships between $\mathrm{C}$ and $\mathrm{CK}$. A critical value of $\mathrm{p}<0.05$ was considered significant.

\section{RESULTS}

The individual values of $\mathrm{C}$ and $\mathrm{CK}$ as well as the coefficients of variation are presented in Tables 1 and 2, respectively.

The number of episodes of hypercortisolaemia varied between 2 and 4 for individual athletes. There were no cases of hypercortisolaemia among 
measurements 1 and 6. Only one player had normal cortisol level during preseason preparation (M2). The highest variability of $\mathrm{C}$ was detected in the first sample, which represents the off-season stress status. As is evident from Table 1 , the intra-individual coefficients of variation for cortisol fit a relatively narrow range.

Table 1. The matrix of cortisol values and coefficients of variation

\begin{tabular}{lccccccc}
\hline & M1 & M2 & M3 & M4 & M5 & M6 & CV $_{\text {intra-individual }}$ \\
\hline A1 & 245 & $\mathbf{7 6 1}$ & $\mathbf{7 9 5}$ & $\mathbf{7 8 4}$ & $\mathbf{7 3 1}$ & 477 & 0.302 \\
\hline A2 & 221 & $\mathbf{8 4 1}$ & 687 & $\mathbf{7 2 6}$ & 681 & 615 & 0.281 \\
\hline A3 & 235 & $\mathbf{8 2 5}$ & 582 & $\mathbf{7 3 9}$ & 579 & 464 & 0.282 \\
\hline A4 & 563 & $\mathbf{1 1 2 6}$ & $\mathbf{7 2 3}$ & $\mathbf{8 0 0}$ & 679 & 560 & 0.365 \\
\hline A5 & 356 & 640 & $\mathbf{8 4 4}$ & $\mathbf{8 0 6}$ & $\mathbf{7 3 9}$ & 538 & 0.339 \\
\hline A6 & 350 & $\mathbf{7 8 6}$ & $\mathbf{7 8 4}$ & 571 & $\mathbf{6 9 8}$ & 439 & 0.353 \\
\hline CV $_{\text {inter-individual }}$ & 0.393 & 0.194 & 0.127 & 0.119 & 0.083 & 0.129 & \\
\hline
\end{tabular}

Cortisol concentrations presented in nmol/l. A1...A6 - athletes from first to sixth. M1...M6 measurements from first to sixth. The values in bold exceed the upper limit $(690 \mathrm{nmol} / \mathrm{l})$ of the reference interval. $\mathrm{CV}$ - coefficient of variation.

Table 2. The matrix of creatine kinase values and coefficients of variation

\begin{tabular}{lccccccc}
\hline & $\mathrm{M} 1$ & $\mathrm{M} 2$ & $\mathrm{M} 3$ & $\mathrm{M} 4$ & $\mathrm{M} 5$ & $\mathrm{M} 6$ & $\mathrm{CV}_{\text {intra-individual }}$ \\
\hline $\mathrm{A} 1$ & $\mathbf{2 7 6}$ & $\mathbf{5 7 6}$ & $\mathbf{7 2 0}$ & $\mathbf{4 1 6}$ & $\mathbf{6 3 5}$ & $\mathbf{2 7 3}$ & 0.392 \\
\hline $\mathrm{A} 2$ & 84 & 541 & $\mathbf{7 7 7}$ & 421 & $\mathbf{1 3 3 4}$ & $\mathbf{5 9 1}$ & 0.667 \\
\hline A3 & 158 & 167 & 264 & 132 & 364 & 136 & 0.453 \\
\hline A4 & 70 & 225 & 184 & 64 & 381 & 182 & 0.632 \\
\hline A5 & 172 & 178 & 500 & 127 & 367 & 184 & 0.573 \\
\hline A6 & 64 & 192 & 272 & 126 & 265 & 195 & 0.432 \\
\hline CV $_{\text {inter-individual }}$ & 0.597 & 0.611 & 0.558 & 0.747 & 0.717 & 0.645 & \\
\hline
\end{tabular}

CK concentrations presented in U/I. A1...A6 - athletes from first to sixth. M1...M6 measurements from first to sixth. The values in bold exceed triple upper limit (190 U/I) of the normal reference interval. $\mathrm{CV}$ - coefficient of variation.

Altogether 21 episodes of elevated CK concentration were detected among 36 measurements. Episodes with the markedly increased amount of $\mathrm{CK}$, exceeding the triple upper limit of the reference interval, were detected only in two athletes (see Table 2).

Altogether 21 episodes of elevated CK concentration were detected among 36 measurements. Episodes with the markedly increased amount of CK, 
exceeding the triple upper limit of the reference interval, were detected only in two athletes (see Table 2).

There was found no direct correlation between individual $\mathrm{C}$ and $\mathrm{CK}$ values with only a slight tendency for one athlete $(r=0.794, p=0.059)$.

Figures 1 and 2 represent the individual dynamics of the analysed variables. As shown in Figure 1, all individual trends follow a similar pattern consistent with the training volume and intensity. An immediate increase in blood $\mathrm{C}$ is evident after the beginning of regular training. Another general trend is the decrease of the stress marker under the influence of tapering.

As shown in Figure 2, intense training throughout the pre-season was associated with muscle damage, but not in all players. The highest CK values were associated with certain bodily collisions.

The number of episodes with a proportional change in the concentration of $\mathrm{C}$ exceeding personal coefficients of variation was different for individual athletes.

As seen from Figure 3, the direction and magnitude of $\mathrm{C}$ response were highly similar in individual athletes after the beginning of regular training. Further on, the uniformity of changes in $\mathrm{C}$ response disappeared. Also the effect of tapering had a unidirectional effect on $\mathrm{C}$ levels but the magnitude of changes exceeded the individual coefficients of variation, but not in all players.

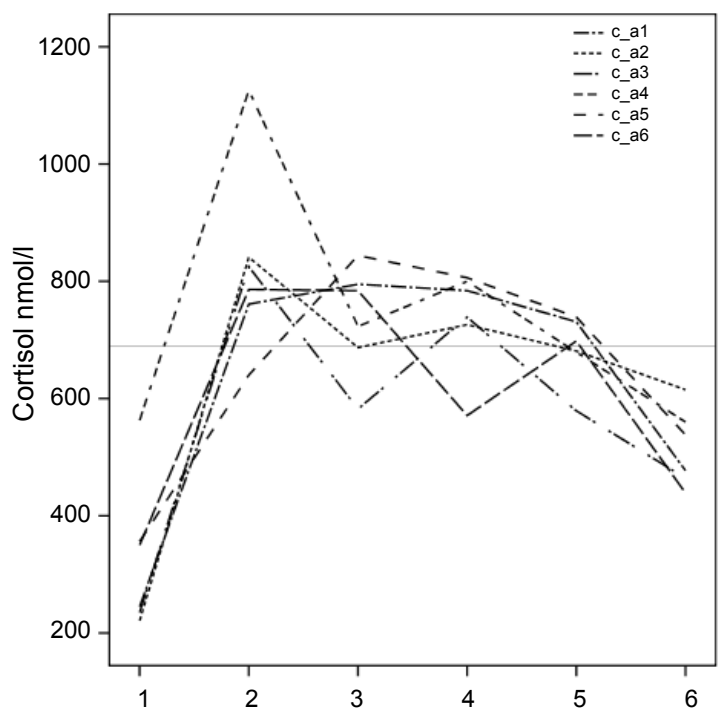

Figure 1. Individual trends of stress response in the basketball players. Upper limit of the normal reference interval $(690 \mathrm{nmol} / \mathrm{l})$ is highlighted as a fine grey line. C - cortisol, a1...a6 - athletes from first to sixth. 


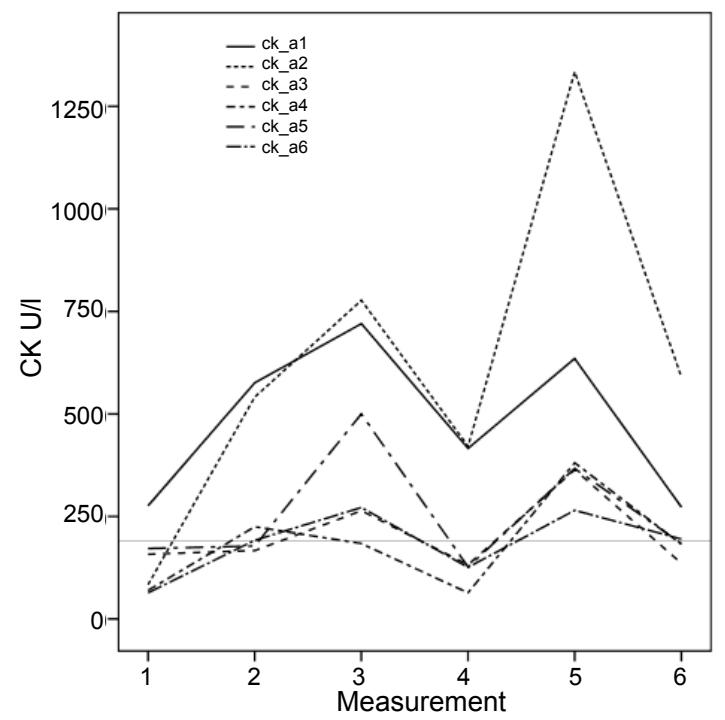

Figure 2. Individual trends of muscle damage in the basketball players. Upper limit of the normal reference interval $(190 \mathrm{nmol} / \mathrm{l})$ is highlighted as a fine grey line. CK - creatine kinase, a1...a6 - athletes from first to sixth.

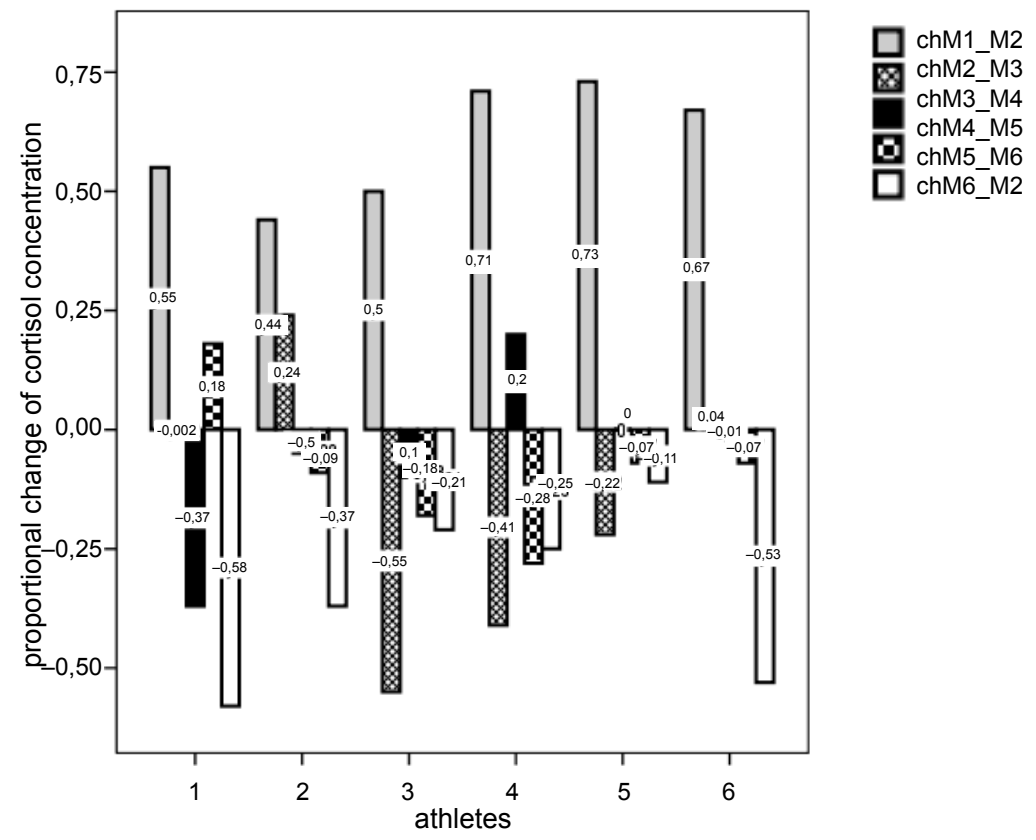

Figure 3. Proportional expression of changes in the level of cortisol throughout the period of observation. 


\section{DISCUSSION}

This study confirms that professional basketball has a temporary effect on body metabolism, resulting in muscle damage and alteration of circulating hormonal levels. The key finding of the study is the observation of repeated episodes of hypercortisolaemia throughout the basketball season, which was clearly associated with the training content. The highest $\mathrm{C}$ levels in the pre-season coincide with the highest training volume in that period. The disappearance of hypercortisolaemia after exercise training requires $2-4$ hours $[7,18]$. Our results suggest a longer period for normalisation of $\mathrm{C}$ level. Blood sampling as extra stress for the investigated athletes cannot be the possible cause of hypercortisolaemia. This postulate is based on the results of the first sampling when no cases of hypercortisolaemia were detected. Our findings are inconsistent with the data of Cunniffe et al. according to which normal levels of $\mathrm{C}$ were detected $14 \mathrm{~h}$ after rugby competition [6]. This inconsistemcy could be explained by differences in the training schedules. Martinez et al. [20] found no hypercortisolaemia in high-level basketball players, either. Different timing regimens for performing blood sampling could be one reason for such a discrepancy between the reported findings about C levels. Kraemer et al. [16] described the predominance of catabolic processes throughout the competitive season in soccer players based on the findings of elevated cortisol level at rest, which is consistent with our findings. An important observation is that the majority of studies dealing with periodical monitoring in athletes are not indicating real base values for pre-season. If the base value is taken from the measurements taken at the preparation period, the results may be impacted not only by the last exercise session, but also by the effects of the whole training micro cycle. Recently it was shown that chronic exposure to intense loads is associated with deprivation of circadian rhythms of cortisol in young athletes [10], which can also explain the revealed discrepancies.

The possible consequences of repeated episodes of hypercortisolaemia 14 hours after the last physical load are not clear. Prolonged hypercortisolaemia is associated with adverse events such as bone demineralization, muscle catabolism, impaired antimicrobial defence, and emotional disturbances. Absence of typical clinical symptoms in the case of above disturbances and concurrent elevated cortisol levels could be explained by limited availability of circulating cortisol described in athletes $[1,7]$. The obtained normal values of $\mathrm{C}$ after 5-day tapering suggest normal reaction of the hypothalamic - pituitary adrenal axis of the involved players. 
Direct comparison of stress as perceived by individual basketball players is possible by the way of calculating the proportional value of $\mathrm{C}$ change between two consecutive measurements.

The analysis of individual proportional changes in C levels revealed mainly two types of response (Fig. 3) to training-induced stress. Some players were able to minimize the high stress response to pre-season preparation during the early in-season. In these players the effect of tapering was less expressed. The favourable effect of tapering on $\mathrm{C}$ level was typical of athletes who were not able to diminish stress response immediately after pre-season preparation. However, more detailed individual analysis requires data about executed physical loads, which was not within the scope of the current study and is also a limitation of the study.

The obtained inter- and intra-individual coefficients of variation support the findings of other similar studies.

Mild persistent increase of $\mathrm{CK}$ is often revealed in regularly exercising persons. The most probable cause of elevated CK level could be occurrence of repeated episodes of eccentric muscle action [2]. Thorpe et al. [23] described clear relationship between number of episodes of highest intensity and muscle damage in soccer. As expected, higher intensity could provoke higher stress and, consequently, higher $\mathrm{C}$ response. Absence of direct correlation between $\mathrm{C}$ and CK can suggest predominance of a low number of responders to CK [2] among involved athletes or a relatively small amount of eccentric activities in basketball. This could be proved using time motion analysis, however such investigation remained beyond the interest of the current study. Our findings regarding elevated CK levels in professional basketball coincide with data from other team ball games $[15,19,23]$. We found no cases of clinically important $\mathrm{CK}$ elevation (>5000 U/L). Body collisions between the basketballs players during a match, which contribute to muscle breakdown, were responsible for the observed episodes of the highest elevation of CK in the current study. The established ranges of intra-individual coefficients of variation of CK were high, which is consistent with results from other studies [22].

It seems that isolated measures of $\mathrm{CK}$ in basketball have a limited practical benefit because of high $\mathrm{CV}$ and the multifactorial background. Expected muscle damage after basketball matches or practices will hopefully stimulate wider use of various methods and modalities to accelerate the recovery of athletes.

Another limitation of this study was that hormonal and biochemical measurements were restricted to a single morning sample and did not cover the 
entire $24 \mathrm{~h}$ cycle, which can be a source of artefacts. Such an intervention design was predicted by the circumstances of the training process as well as by the intention to minimize the possible influence of interventions on the training regimen.

In summary, the findings in the present study confirm that long-term intensive exercise in basketball has a significant influence on the blood cortisol profile. However, stress response and muscle damage were not directly related in the investigated players.

Longitudinal evaluation of fluctuations in blood $\mathrm{C}$ requires a new, individualised approach in assessment of stress and muscle damage, taking account of perceived loads throughout practices and competitions.

\section{REFERENCES}

1. Bonifazi M., Mencarelli M., Fedele V., et al. (2009). Glucocorticoid receptor mRNA expression in peripheral blood mononuclear cells in high trained compared to low trained athletes and untrained subjects. J Endocrinol Invest, 32(10), 816-20.

2. Brancaccio P., Maffulli N., Limongelli F. M. (2007). Creatine kinase monitoring in sport medicine. Br Med Bull, 81-82, 209-30.

3. Córdova A., Sureda A., Tur J. A., Pons A. (2010). Immune response to exercise in elite sportsmen during the competitive season. J Physiol Biochem, 66(1), 1-6.

4. Coutts A.J., Reaburn P., Piva T. J., Rowsell G. J. (2007) Monitoring for overreaching in rugby league players. Eur J Appl Physiol, 99(3), 313-24.

5. Crewther B. T., Cook C., Cardinale M., et al. (2011). Two emerging concepts for elite athletes: the short-term effects of testosterone and cortisol on the neuromuscular system and the dose-response training role of these endogenous hormones. Sports Med, 41(2), 103-23.

6. Cunniffe B., Hore A. J., Whitcombe D. M., et al. (2010). Time course of changes in immuneoendocrine markers following an international rugby game. Eur J Appl Physiol, 108(1), 113-22.

7. Duclos M., Guinot M., Le Bouc Y. (2007). Cortisol and GH: odd and controversial ideas. Appl Physiol Nutr Metab, 32(5), 895-903.

8. Eliakim A., Portal S., Zadik Z., et al. (2009). The effect of a volleyball practice on anabolic hormones and inflammatory markers in elite male and female adolescent players. J Strength Cond Res, 23(5), 1553-9.

9. Fragala M. S., Kraemer W. J., Denegar C. R., et al. (2011). Neuroendocrine-immune interactions and responses to exercise. Sports Med, 41(8), 621-39.

10. Georgopoulos N. A., Rottstein L., Tsekouras A., et al. (2011). Abolished circadian rhythm of salivary cortisol in elite artistic gymnasts. Steroids, 76(4), 353-7. 
11. Gocentas A. (2011). Longitudinal changes of anabolic/catabolic balance in highlevel male basketball. In: $7^{\text {th }}$ EFSMA - European Congress of Sports Medicine, $3^{\text {rd }}$ Central European Congress of Physical Medicine and Rehabilitation. Salzburg, 26.-29.10.2011. Düsseldorf: German Medical Science GMS Publishing House. Doc11esm089.

12. Hackney A. C., Viru A. (2008). Research methodology: endocrinologic measurements in exercise science and sports medicine. J Athl Train, 43(6), 631-9.

13. Handziski Z., Maleska V., Petrovska S., et al. (2006). The changes of ACTH, cortisol, testosterone and testosterone/cortisol ratio in professional soccer players during a competition half-season. Bratisl Lek Listy, 107(6-7), 259-63.

14. Hartwig T. B., Naughton G., Searl J. (2009). Load, stress, and recovery in adolescent rugby union players during a competitive season. J Sports Sci, 27(10), 108794.

15. Ispirlidis I., Fatouros I. G., Jamurtas A. Z., et al. (2008). Time-course of changes in inflammatory and performance responses following a soccer game. Clin J Sport Med, 18(5), 423-31.

16. Kraemer W. J., French D. N., Paxton N. J., et al. (2004). Changes in exercise performance and hormonal concentrations over a big ten soccer season in starters and nonstarters. J Strength Cond Res, 18(1), 121-8.

17. Kraemer W. J., Ratamess N. A. (2005). Hormonal responses and adaptations to resistance exercise and training. Sports Med, 35(4), 339-61.

18. Kraemer W. J., Spiering B. A., Volek J. S., et al. (2009). Recovery from a national collegiate athletic association division I football game: muscle damage and hormonal status. J Strength Cond Res, 23(1), 2-10.

19. Lazarim F. L., Antunes-Neto J. M., da Silva F. O., et al. (2009). The upper values of plasma creatine kinase of professional soccer players during the Brazilian $\mathrm{Na}$ tional Championship. J Sci Med Sport, 12(1), 85-90.

20. Martínez A. C., Seco Calvo J., Tur Marí J. A., et al. (2010). Testosterone and cortisol changes in professional basketball players through a season competition. $\mathrm{J}$ Strength Cond Res, 24(4), 1102-8.

21. Meeusen R., Nederhof E., Buyse L., et al. (2010). Diagnosing overtraining in athletes using the two-bout exercise protocol. Br J Sports Med, 44(9), 642-8.

22. Nunes L. A., Brenzikofer R., de Macedo D. V. (2010). Reference change values of blood analytes from physically active subjects. Eur J Appl Physiol, 110(1), 191-8.

23. Thorpe R., Sunderland C. (2011). Muscle damage, endocrine and immune marker response to a soccer match. J Strength Cond Res, Nov 23. In press.

24. Viru A. (1992). Plasma hormones and physical exercise. Int J Sports Med, 13, 201-209. 


\section{Address for correspondence:}

Anatoli Landõr

University of Tartu

Faculty of Medicine

Ravila 19, Tartu, 50406, Estonia

E-mail: anatolilandor@gmail.com 